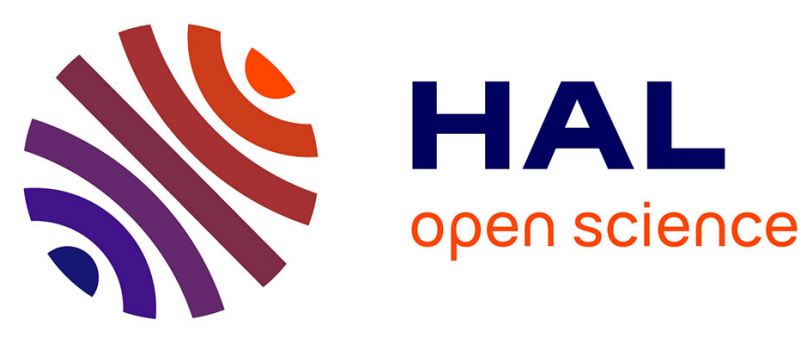

\title{
Power domination in graphs
}

Paul Dorbec

\section{To cite this version:}

Paul Dorbec. Power domination in graphs. Topics in Domination in Graphs, 2020, 978-3-030-51117-3. 10.1007/978-3-030-51117-3_16. hal-03073783

\section{HAL Id: hal-03073783 \\ https://hal.science/hal-03073783}

Submitted on 16 Dec 2020

HAL is a multi-disciplinary open access archive for the deposit and dissemination of scientific research documents, whether they are published or not. The documents may come from teaching and research institutions in France or abroad, or from public or private research centers.
L'archive ouverte pluridisciplinaire HAL, est destinée au dépôt et à la diffusion de documents scientifiques de niveau recherche, publiés ou non, émanant des établissements d'enseignement et de recherche français ou étrangers, des laboratoires publics ou privés. 


\title{
Power domination in graphs
}

Paul Dorbec

\begin{abstract}
In this chapter, we are interested in power domination in graphs. Power domination is a variation of domination introduced to address a physical problem of monitoring a network with phasor measurement units. The originality of this variation is that some propagation happens, and the set of covered vertices results from an iterative process. We present a survey of known results on this specific parameter.
\end{abstract}

\section{Introduction}

Power domination is a variation of the domination problem motivated by the physical rules for monitoring electrical networks. It was first introduced as a graph parameter by Haynes et al. in 2002 [20]. Before giving details on the initial motivation and the physical background in Section 1.1, we give a general definition. One of the key concept of power domination is that of monitoring vertices, as defined below.

\section{Definition 1 (Power dominating set)}

Given a graph $G=(V, E)$ and a set $S \subseteq V$ of vertices, we define the set of vertices monitored by the set $S$ as follows:

- Domination: All vertices in $S$ and all neighbors of vertices in $S$ are monitored,

- Propagation: Whenever a vertex $v$ is monitored and all but one of its neighbors, say $w$, are monitored, then vertex $w$ is also monitored. In this case we say that vertex $v$ propagates to vertex $w$.

An initial set of vertices that eventually monitors the whole graph is called a power dominating set. The power domination number is the minimum order of a power dominating set, denoted $\gamma_{P}(G)$.

Paul Dorbec

Normandie Univ, UNICAEN, ENSICAEN, CNRS, GREYC, 14000 Caen, France, e-mail: paul.dorbec@unicaen.fr 
The first step, the so called domination step, exactly matches the definition of a dominating set. Thus a dominating set is also a power dominating set, and we observe that

$$
\forall G, \quad \gamma_{P}(G) \leq \gamma(G)
$$

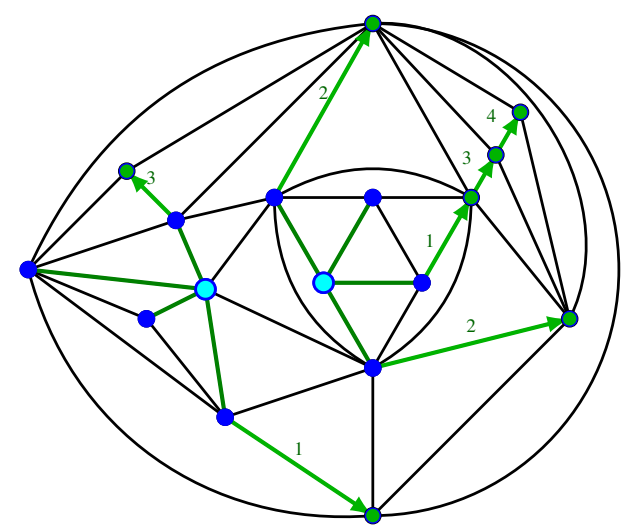

Fig. 1 A graph with a power dominating set (in Cyan). The arrows show the propagation occurring, and their labels gives the ordering of these propagation.

An example of a graph with a power dominating set is shown in Figure 1. The difference between the usual domination and power domination is best illustrated by the case of the path. Indeed, the power domination number of a path is equal to 1 , and any vertex is a minimum power dominating set of the path. This comes from the fact that after the initial step of domination in the path, every monitored vertex has at most one unmonitored neighbor, and thus can propagate. So the set of monitored vertices increases until it covers the whole path.

Before continuing, we present a more formal definition that sometimes proves convenient. We define the sets $\left(\mathcal{P}^{i}\right)_{i \geq 0}$ of monitored vertices at stage $i$, following the definition introduced by Aazami in [1].

\section{Definition 2 (Monitored vertices)}

Let $G$ be a graph, and $S \subseteq V(G)$ a subset of vertices. We define the sets $\left(\mathcal{P}^{i}(S)\right)_{i \geq 0}$ of vertices monitored by $S$ at step $i$ by the following rules.

- Domination: $\mathcal{P}^{0}(S)=N[S]$,

- Propagation: for $i \geq 0$,

$$
\mathcal{P}^{i+1}(S)=\bigcup\left\{N[v]\left|v \in \mathcal{P}^{i}(S),\right| N[v] \backslash \mathcal{P}^{i}(S) \mid \leq 1\right\} .
$$

Observe that the sequence of sets $\left(\mathcal{P}^{i}\right)_{i \geq 0}$ is a non decreasing sequence. Moreover if at some stage $\mathcal{P}^{i}(S)=\mathcal{P}^{i+1}(S)$, then the sequence reaches a fixed point, which we 
denote $\mathcal{P}^{\infty}(S)$. Observe that the fixed point is necessarily reached for some $i<n$, so for a graph on $n$ vertices, $\mathcal{P}^{\infty}(S)=\mathcal{P}^{n}(S)$. Matching the earlier definition, we can now state that $S$ is a power dominating set of a graph $G$ if and only if $\mathcal{P}^{\infty}(S)=V(G)$.

With this notation, for a vertex $u$ in a path, $\mathcal{P}^{i}(\{u\})=\{v \in V \mid \operatorname{dist}(u, v) \leq i+1\}$. In the example from Figure 1, the label $i$ on an arc shows that a vertex is first added to $\mathcal{P}^{i}(S)$.

\subsection{Physical motivation}

In this section, we recall the history of the introduction of the parameter, with the successive definitions.

Power domination was first introduced by Baldwin et al. in [3], then described as a graph theoretical problem by Haynes et al. in [20]. The problem is motivated by the requirement for constant monitoring of power systems by placing a minimum number of phasor measurement units (PMU) in the network. A PMU placed at a bus measures the voltage of the bus plus the current phasors at that bus. Using Ohm and Kirchhoff current laws, it is then possible to infer from initial knowledge of the status of some part of the network the status of new branches or buses.

In Baldwin et al. [3], the following definitions are proposed:

A measurement-assigned subgraph, called for short a measurement subgraph, is a subgraph which has a current measurement assigned to each of its branches. These are either actual measurement or calculated pseudo-measurement deduced from Kirchhoff's and Ohm's laws.

[...] The coverage of a placement set of PMU's is the maximal spanning measurement subgraph that can be formed by this set, that is, the maximal observable sub-network that can be built from them.

They introduced the following formal definition of the spanning measurement subgraph:

\section{Definition 3 (Baldwin et al. [3])}

A spanning measurement subgraph is constructed throughout the network on the grounds of the following rules:

Rule 1: Assign a current phasor measurement to each branch incident to a bus provided with a PMU;

Rule 2: Assign a pseudo-current measurement to each branch connecting two buses with known voltage;

Rule 3: Assign a pseudo-current measurement to a branch whose current can be inferred by using Kirchhoff's current law.

In terms of graphs, where buses are vertices and connecting branches are edges, we can describe the observability rules of a network with the following definition:

Definition 4 (Haynes et al. [20]) 
Initially, set as monitored any vertex with a PMU and all edges incident to it. Then, expand iteratively the set of monitored edges and vertices with the following rules :

1. set as monitored any vertex incident to a monitored edge whose other end is monitored;

2. set as monitored any edge joining two monitored vertices;

3. if a vertex has all its incident edges monitored except one, set this one edge as monitored.

It was noticed by Dorfling and Henning in [15] that the power domination problem can be studied considering only vertices following the above definition. The coverage of a placement set $S$ of PMU is then simply the induced subgraph on the final set of monitored vertices. From this observation, we reach the definition used here, presented in Definition 1.

\subsection{Relation with zero forcing sets}

It should be noted that there is a close relationship between power domination in graphs and zero forcing sets. Zero forcing sets were introduced in [2], together with the corresponding parameter $Z(G)$, which stands for the minimum size of a zero forcing set. Using the earlier definition, we can define a zero forcing set as a set of vertices $S$ such that applying only the propagation rule, the whole graph eventually gets monitored. In other words, it would follow Definition 2 where we define $\mathcal{P}^{0}(S)$ to be equal to $S$.

The motivation for the introduction of this parameter was that it is an upper bound for another parameter, called maximum nullity of a graph. For a graph $G$ of order $n$, the maximum nullity of $G$ corresponds to the maximum nullity (or corank) of a matrix in the set of symmetric $n \times n$ matrices having nonzero coefficients precisely where the adjacency matrix of the graph $G$ has nonzero values.

From the above definition of zero forcing sets, we easily infer that $Z(G) \leq$ $\gamma_{P}(G) \Delta(G)$. Indeed, taking the vertices of a power dominating set plus all but one neighbor of each of them, one gets a zero forcing set of size at most $\gamma_{P}(G) \Delta(G)$. This was explicitly stated by Dean et al. in [9], where the first link between the two parameters was probably made.

Various later studies considered or just mentioned the link between zero forcing sets and power domination $[7,16,5]$, or even between $k$-forcing sets and $k$-power domination that we define in Section 5 [17]. However, there are not many results for power domination that come from known results on zero forcing sets. 


\subsection{Algorithmic aspects}

We will not detail the algorithmic aspects here, since it is quite similar to the domination algorithms, and it is surveyed in the chapter dedicated to algorithms. It should just be remarked that power domination is NP-complete, with many possible reductions from the dominating set problem. However, as for the domination problem, there are polynomial algorithms for bounded treewidth graphs, using dynamic programming. The algorithms are slightly more involved than for domination, but use similar strategies.

\section{Behaviour by small graph changes}

As we explore in this section, one of the difficulties of power domination is that the power domination number has no monotonicity for any of the classical graph operations. For each of the usual unitary graph operations (vertex removal, edge removal, edge contraction), we give some examples and arguments illustrating the possible behavior of the power domination number. Detailed proofs of these results were given by Dorbec, Varghese and Vijayakumar in [14].

\subsection{Vertex removal}

In a graph, the removal of a vertex can have a similar effect on the power domination number than it can have on the domination number, that is that it can slightly reduce the power domination number, or it can much increase it.

Theorem 1 ([14]) For the graph $G-v$ obtained by removing a vertex $v$ from a graph $G$, there is no upper bound to $\gamma_{P}(G-v)$ in terms of $\gamma_{P}(G)$. On the other hand, we have $\gamma_{P}(G-v) \geq \gamma_{P}(G)-1$.

As in domination, adding $v$ to a power dominating set of $G-v$ produces a power dominating set of $G$. On the other hand, removing the central vertex of a star greatly increases the power domination number. Less trivial examples are given in [14].

\subsection{Edge removal}

Interestingly, the situation for edge removal is not as similar to the situation for the dominating sets. While removing an edge in a graph can only increase its domination number, it may decrease its power domination number.

Theorem 2 ([14]) Removing an edge e from a graph $G$ results in a graph $G-e$ whose power domination number is bounded by 


$$
\gamma_{P}(G)-1 \leq \gamma_{P}(G-e) \leq \gamma_{P}(G)+1 .
$$

That removing an edge may increase the power domination number is of no surprise, as it is for domination. For a removed edge $e=u v$ in a graph $G$ with a power dominating set $S$, if $v$ is monitored no sooner than $u$ in the graph $G$, then $S \cup\{v\}$ is a power dominating set of $G-e$.

However, that removing an edge may also decrease the power domination number is less expected. Actually, this phenomenon comes from the fact that the removal may allow some propagation that was not possible before, as in the example of the bipartite complete graph $K_{3,3}$ (see Figure 2), but also for many other examples.

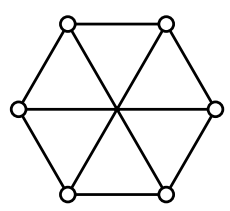

Fig. 2 The graph $K_{3,3}$ has power domination number 2, removing any edge decrease this to 1 .

\subsection{Edge contraction}

Contracting an edge in a graph may easily result into the reduction of the domination number by one, the same is true for the power domination number. This happens for example when a minimum (power) dominating set contains both ends of the contracted edge, as in a double star. What happens in power domination but not in domination is that it may also increase the power domination number.

Theorem 3 ([14]) Let $G$ be a graph and e be an edge in $G$. Then

$$
\gamma_{P}(G)-1 \leq \gamma_{P}(G / e) \leq \gamma_{P}(G)+1 .
$$

An (original) example where the power domination number of a graph increases when an edge is contracted is drawn in Figure 3. In this graph, the contraction of the edge $e$ merges two vertices with only one unmonitored neighbor, and thus prevents propagation.

To show that the power domination number increases by no more than one, just note that adding the newly formed vertex to a power dominating set of $G$ necessarily forms a power dominating set of $G / e$, preventing the previous phenomenon. 


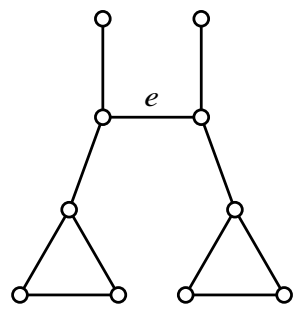

Fig. 3 A graph where contracting the edge $e$ makes the power domination number increase from 2 to 3 .

\section{Bounds on general families of graphs}

In this section, we present bounds on the power domination number of a graph under some general restrictions.

\subsection{General graphs}

Let us first recall the initial general bound due to Haynes et al. [20]. They noted that the power domination number of a graph is always at least one, and that a dominating set of a graph is always also power dominating. We thus get

$$
1 \leq \gamma_{P}(G) \leq \gamma(G) .
$$

Haynes et al. proved that there is no forbidden subgraph characterization of the graphs reaching the upper bound. The proof is based on the following family of graphs. For any graph $G$, take the graph family $\mathcal{T}(G)$ of the graphs obtained by adding for each vertex $v \in V(G)$ two new vertices, namely $v_{0}$ and $v_{1}$, with the edges $v v_{0}$ and $v v_{1}$, and possibly or not the edge $v_{0} v_{1}$ (see Figure 4).

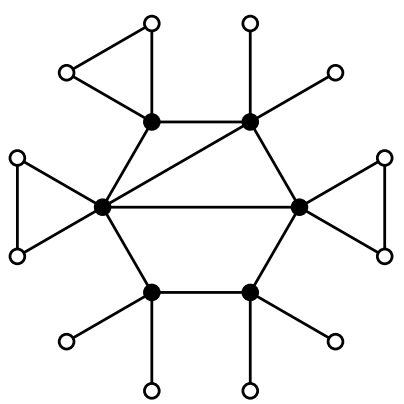

Fig. 4 A graph from the family $\mathcal{T}$ built on a six vertices initial graph (on black vertices). 
For all such graphs $G^{\prime} \in \mathcal{T}(G), \gamma_{P}\left(G^{\prime}\right)=\gamma\left(G^{\prime}\right)=|V(G)|=\frac{\left|V\left(G^{\prime}\right)\right|}{3}$. Now, observing that the initial graph $G$ may be any graph proves the above statement.

Actually, Zhao, Kang and Chang proved in [32] that this construction plays a special role, while proving the following general bound. We denote by $\mathcal{T}$ the union of $\mathcal{T}(G)$ over all graphs $G$.

Theorem 4 (Zhao, Kang, Chang [32]) For any connected graph $G$ of order $n \geq 3$, $\gamma_{P}(G) \leq n / 3$ with equality if and only if $G \in \mathcal{T} \cup\left\{K_{3,3}\right\}$.

Note that this bound is an improvement of the same bound proved only for trees by Haynes et al. in [20].

To prove this result, they first recall (from [20]) that in any connected graph with maximum degree at least 3 , there exists a minimum power dominating set containing only vertices of degree at least 3 . Then, they show that the set $S$ can be chosen so that every vertex in $S$ has at least two private neighbors (by minimizing the number of edges in $G[S]$ ). The result follows.

This result can be seen as a generalization of the $\frac{n}{2}$ bound for domination, and the constructions look similar. Interestingly, the relationship between these two bounds is even more enlightened for generalized power domination, as observed in Section 5.

In [3], Baldwin et al. conjectured an upper bound on the size of a power dominating set. They considered the possibility of unknown power injections, which in graphs could be seen as a single leaf attached to the corresponding vertex/bus. That explains the unusual expression of the conjecture:

Conjecture 1 (Baldwin et al. [3])

Let $G$ be a graph on $n$ vertices of which $k$ are of degree one. If no vertex of $G$ is adjacent to more than one leaf, then the power domination number of $G$ satisfies

$$
\gamma_{P}(G) \leq\left\lceil\frac{2 n-k}{6}\right\rceil .
$$

To illustrate the conjecture and prove that this bound is tight if correct, they present the graph depicted in Figure 5. Note that though the above theorem proves it for graphs with no degree 1 vertex, the conjecture does not seem to have been considered on its own elsewhere.

\subsection{Regular graphs}

For regular graphs, it seems that better bounds can be proved. Zhao, Kang, Chang [32] got the first results in that direction, using as an additional condition that the graph is claw-free. A few years later, Dorbec et al. proved in [11] the same bound, dropping the claw-free condition, but still excluding the only known counter-example, which is $K_{3,3}$. 


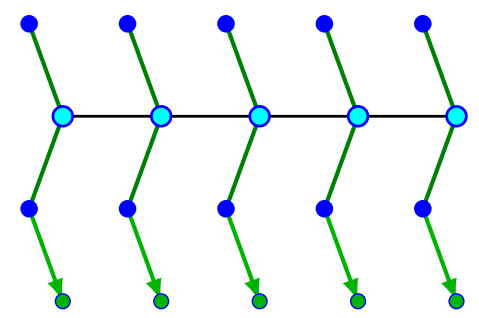

Fig. 5 A graph attaining the bound of Conjecture 1. Black vertices form a minimum power dominating set.

\section{Theorem 5 (Dorbec et al. [11])}

Let $G$ be a connected cubic graph on $n$ vertices. If $G$ is not the complete bipartite graph $K_{3,3}$, then $\gamma_{P}(G) \leq \frac{n}{4}$.

The bound is known to be tight thanks to the example of Zhao, Kang, Chang [32], consisting in a set of $K_{4}$ minus an edge, using the degree 2 vertices of $K_{4}-e$ to join them into a cycle (see Figure 6). A minimum power dominating set is obtained by choosing one vertex of degree three from each of the six subgraphs $K_{4}-e$.

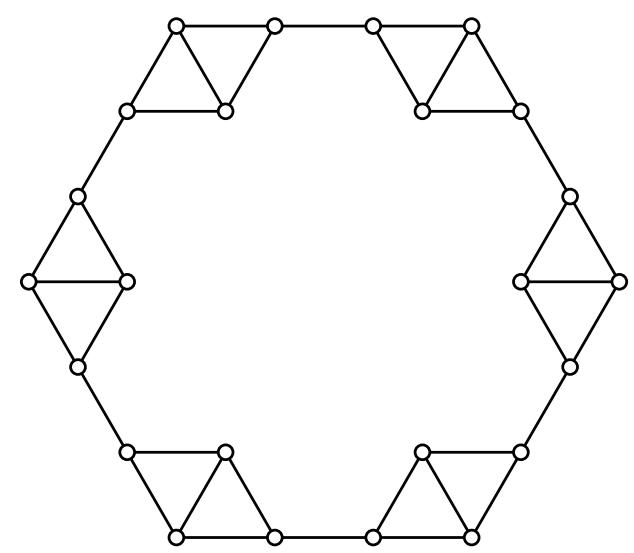

Fig. 6 An example of a cubic graph reaching the bound of Theorem 5.

The proof of this result is quite technical, but the idea behind the proof may be re-used. The strategy was, for a given initial set of vertices, to study what may happen at the boundary of the set of monitored vertices (the peripheral vertices). That the set does not continue to propagate gives quite some information (in particular in a cubic graph). Then one is likely to find a vertex whose addition to the set of selected vertices would greatly increase the number of monitored vertices, maintaining the expected bound. Structures that would prevent this are very special, and are retrospectively dealt with during the initial choice of the set $S$ of vertices. 
Recently, Kang and Wormald [21] studied the power domination number on random cubic graphs. They proved that the power domination number of a random cubic graph of order $n$ is asymptotically almost surely between $0.033 n$ and $0.068 n$.

\subsection{Maximal planar graphs}

Among the general bounds on the power domination number of a graph, there is a recent result on maximal planar graphs, by Dorbec, Gonzales and Pennarun [10]. Applying a technique similar to the one for cubic graphs, Dorbec, Gonzales and Pennarun [10] proved that maximal planar graphs satisfy the following inequality:

Theorem 6 (Dorbec, Gonzales, Pennarun [10]) Every maximal planar graph $G$ on $n$ vertices has a power domination number at most:

$$
\gamma_{P}(G) \leq \frac{n-2}{4} .
$$

The known graphs for which this bound is tight have at most 10 vertices (see Figure 7 for the largest known example). This leads one to think that a better bound should exist. Until now, the maximal planar graphs having the largest known power domination number are obtained from a disjoint set of octahedra between which are added edges until reaching a maximal planar graph. A vertex specific to each octahedron is required to dominate such a graph, and thus this graph has power domination number $\frac{n}{6}$.

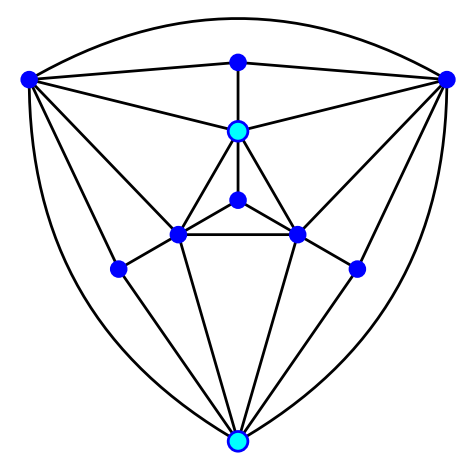

Fig. 7 The triakis tetrahedron, a maximal planar graph on 10 vertices with power domination number 2 .

This is similar to the known bounds for the domination number of maximal planar graphs, the worst known family being maximal planar graphs obtained by adding edges between a set of disjoint $K_{4}$, which implies a domination number $\frac{n}{4}$, for an upper bound on the domination number of maximal planar graphs being at most $\frac{n}{3}$. 


\subsection{Bounded diameter graphs}

Several attempts have been made to bound the power domination number in terms of the diameter of a graph. It was noted by Zhao and Kang that planar graphs with diameter at most 2 have a power domination number at most 2. However, they left as an open question whether the power domination number of a graph of diameter at most 2 could be large.

Soh and Koh proposed in [26] families of graphs with diameter at most 2 and unbounded power domination number. One family is simply the Cartesian product $K_{n} \square K_{m}$ of two complete graphs:

Theorem 7 (Soh and Koh [26]) For any $m \geq n \geq 2$, $\gamma_{P}\left(K_{n} \square K_{m}\right)=n-1$.

The other is an infinite graph, called the Rado graph, whose vertices are labelled with integers. In this graph, two vertices $x<y$ are adjacent if and only if the $x^{\text {th }}$ digit in the binary representation of $y$ is 1 . Any $x$ and $y$ have as a common neighbor $\left(2^{x}+2^{y}\right)$, which implies that the Rado graph has diameter 2 . However, no propagation is possible in that graph since every vertex has infinitely many neighbors. Thus, the Rado graph has the same domination number and power domination number, and no finite subset of this graph is a dominating set. Note that the argument does not apply though to finite subgraphs of the Rado graph.

\section{Recursively defined families}

In this section, we consider the graph families for which explicit formulas are known.

Generally, when searching for a power dominating set of a graph, it is not too difficult to figure out what seems to be a good selection of vertices. This usually gives a pretty good upper bound to the power domination number of the graph. However, finding lower bounds turns out to be quite hard in general. In the following, we thus get into quite some details when original techniques are used to prove lower bounds.

To start with, a nice statement is that each vertex may be used for propagation only once, when it has exactly one unmonitored neighbor. Let a peripheral vertex be a monitored vertex with at least one unmonitored neighbor. What the earlier remark enables one to infer is that during the process of propagation, the number of peripheral vertices cannot increase. So we get as an invariant property that the number of peripheral vertices in the graph is no more than the sum of the degrees of the vertices in the initial set $S$. This was explicitly stated in [24], though it was implicitly used before. Though this seems a good handle to provide lower bounds, what makes this invariant not so easy to use is that at the end of the propagation steps, there are no peripheral vertices left. However, some of the later proofs show variants of this notion of peripheral vertices that are useful. 


\subsection{Products and grids}

In this section, we consider results on power domination in graph products, and in particular on the Cartesian product. We also consider other lattices, such as hexagonal and triangular grids.

It should be noted that a recent survey of results on graph products is given by Soh and Koh in [27], which is more detailed than what we present here, and should be referred to for having an exhaustive list of theorems on the topic. The same authors also surveyed earlier the results on the Cartesian product in [22].

\subsubsection{Cartesian product}

Power domination in products of paths were among the first topics to be studied on power domination. Dorfling and Henning [15] studied the Cartesian product of two paths, i.e. grid graphs.

Theorem 8 (Dorfling, Henning [15]) The power domination number of the $n \times m$ grid $P_{n} \square P_{m}$ for $m \geq n \geq 1$ is

$$
\gamma_{P}\left(P_{n} \square P_{m}\right)= \begin{cases}\left\lceil\frac{n+1}{4}\right\rceil & \text { if } n \equiv 4 \quad(\bmod 8), \\ \left\lceil\frac{n}{4}\right\rceil & \text { otherwise. }\end{cases}
$$

In their proof, they explicitly describe the shape of the set of monitored vertices by any initial subset of vertices in the grid. In addition, their proof also relies on a study of the cylinder, using the number of 'columns' as an invariant, though, the use of an invariant is not explicit. The question on the cylinder (i.e. the product of a path and a cycle) was also studied later by Barrera and Ferraro [4] as well as the torus (product of two cycles).

The hypercube is also an interesting graph family for studying power domination. Actually, in the graph $G \square K_{2}$, dominating one copy of $G$ is enough to power dominate the whole graph $G \square K_{2}$. Therefore, we get that $\gamma_{P}\left(G \square K_{2}\right) \leq \gamma(G)$ for any graph $G$. For the hypercube, this was observed by Dean et al. in [9]. They further conjectured that the domination number of $Q_{n}$ was equal to the power domination number of $Q_{n+1}$. But later on, Pai and Chiu [25] showed that $\gamma\left(Q_{5}\right)=7$ while $\gamma_{P}\left(Q_{6}\right)=6$, disproving their conjecture.

More results are proved by Varghese and Vijayakumar in [28] and by Soh and Koh in [27], in particular towards a characterization of graph products having power domination number equal to one. We refer the reader to the survey [27] for more details.

\subsubsection{Strong product}

The study on the products of paths was continued by Dorbec, Mollard, Klavžar and Špacapan in [13] with the three other classical products. 
For the strong product, they prove the following lower bound

Theorem 9 (Dorbec et al. [13]) Let $m \geq n \geq 2$. Then

$$
\gamma_{P}\left(P_{m} \otimes P_{n}\right) \geq \max \left\{\left\lceil\frac{m}{3}\right\rceil,\left\lceil\frac{m+n-2}{4}\right\rceil\right\}
$$

Whenever $3 n-m-6 \not \equiv 4(\bmod 8)$, one can construct an initial set $S$ which achieves this lower bound. For the remaining case, Soh and Koh claimed in [27] to have proved that the correct value is $\left\lceil\frac{m+n-2}{4}\right\rceil+1$ though their proof is missing some details.

An interesting aspect of this lower bound is that it is proved by considering not just the number of peripheral vertices, but the number of non-surrounded vertices, that is of monitored vertices having at most seven monitored neighbors (recall that the maximal number of neighbors in the strong product of two paths is eight). This includes all peripheral vertices, but also the vertices from the border of the grid. The first step of the proof is then that the same invariant property can be proved for non surrounded vertices, under some conditions (by proving that any propagation that would start on a vertex from the border could have been made from another vertex too). This invariant enables one to prove this lower bound, based on the number of vertices on the border of the grid.

Again, Soh and Koh in [27] claim the same results for the product of cycle, based on the same proof, but they do not explain how they adapt the invariant.

\subsubsection{Direct product}

For the direct product of paths (which has two connected components), the first bound obtained in [13] can be stated as follows:

Theorem 10 ([13]) The power domination number of the direct product $P_{n} \times P_{m}$ for $m \geq n \geq 1$ is

$$
\gamma_{P}\left(P_{n} \times P_{m}\right)=\left\{\begin{array}{l}
2\left\lceil\frac{n}{4}\right\rceil \text { if } n \text { is even, } \\
2\left\lceil\frac{m}{4}\right\rceil \text { if } n \text { is odd and } m \text { even, }
\end{array}\right.
$$

If both $m$ and $n$ are odd,

$$
\gamma_{P}\left(P_{n} \times P_{m}\right) \leq \max \left\{\left\lceil\frac{m}{4}\right\rceil+\left\lceil\frac{m-2}{4}\right\rceil,\left\lceil\frac{m+n}{6}\right\rceil+\left\lceil\frac{m+n-2}{6}\right\rceil\right\}
$$

Actually, it is proved that one component has power domination number exactly $\max \left\{\left\lceil\frac{m}{4}\right\rceil,\left\lceil\frac{m+n}{6}\right\rceil\right\}$ whereas for the other component, the only lower bound proved is $\frac{n}{4}$. The proof is very technical, using a connection with a percolation process on a square grid as a reference.

The case of the product of two cycles was also considered in [27], who mentioned some earlier communications in a workshop on the topic. They also use the notion of peripheral vertices (there called boundary vertices), with a nice trick. Their idea 
is to take a minimum power dominating set $S$ of the graph, remove one vertex $v$ from it, and then count the number of boundary vertices for $\mathcal{P}^{\infty}(S \backslash\{v\})$.

\subsubsection{Lexicographic product}

The case of the lexicographic product is easier, since the role of the graph $G$ is much more important than the role of $H$ in $G \circ H$. Actually, unless the power domination number of $H$ is one, it is as good to totally dominate $G \circ H$ than to use propagation.

Theorem 11 ([13]) For any nontrivial graphs $G$ and $H$, if $G$ has no isolated vertices, then

$$
\gamma_{P}(G \circ H)=\left\{\begin{aligned}
\gamma(G) ; \gamma_{P}(H) & =1 \\
\gamma_{t}(G) ; \gamma_{P}(H) & >1
\end{aligned}\right.
$$

\subsubsection{Hexagonal grids and triangular grids}

The first results on the hexagonal grid are given by Ferrero, Varghese ${ }^{1}$ and Vijayakumar in [18]. They consider hexagonal grids with an hexagonal outer shape and give the exact domination number of those grids. The method used is related to the method of Dorfling and Henning in [15] for the square grid.

Theorem 12 (Ferrero, Varghese, Vijayakumar [18]) Let HM(n) be the hexagonal grid with an hexagonal outer shape whose side is made of $n$ hexagons. We have

$$
\gamma_{P}(H M(n))=\left\lceil\frac{2 n}{3}\right\rceil .
$$

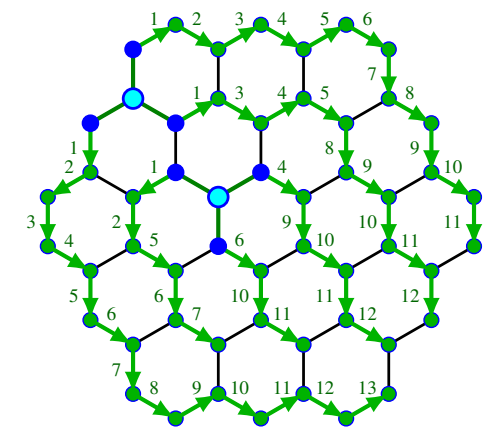

Fig. 8 The hexagonal grid $H M(3)$.

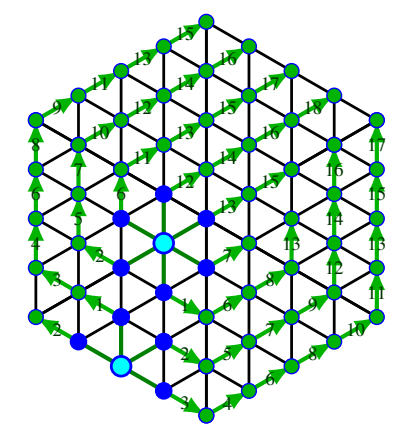

Fig. 9 The triangular grid $T_{5}$.

${ }^{1}$ Seema Varghese who is cited here, is actually the elder sister of Seethu Varghese who was cited elsewhere. Both of them were PhD students of Vijayakumar. 
When studying the triangular grid with hexagonal outer shape, Bose, Pennarun and Verdonschot [6] noticed a conection with that earlier result. They got a similar bound:

Theorem 13 (Bose, Pennarun, Verdonschot [6]) For $k \geq 1$, le $T_{k}$ be the triangular grid with hexagonal shape, whose side is made of $k$ vertices. We have

$$
\gamma_{P}\left(T_{k}\right)=\left\lceil\frac{k}{3}\right\rceil .
$$

However, to show the lower bound, they used a very different technique than Ferrero, Varghese and Vijayakumar. Their nice idea is to consider a projection of the mogreyc-amacc:nitored set on one side, and then count the number of peripheral vertices of that projection when about half the vertices are monitored. They prove that this second number is a lower bound to the number of peripheral vertices before the projection. This results in a very original way of using the peripheral vertices invariant.

\subsection{Other Recursively defined families}

In this section, we survey the main recursively defined families for which the power domination number has been computed.

\subsubsection{Generalized Petersen graphs and permutation graphs.}

The case of generalized Petersen graphs was considered by both Barrera and Ferrero in [4] and by $\mathrm{Xu}$ and Kang in [31]. In [4], they suggest a more general study on Cayley graphs as a continuation of this study.

Actually, the work was continued on permutation graphs which form a superfamily of generalized Petersen graphs. Those were considered by Wilson in [30]. In his paper, Wilson mainly conjectured that in a permutation graph $G$ on $n$ vertices (that is based on two cycles on $\frac{n}{2}$ vertices), $\gamma_{P}(G) \leq\left\lceil\frac{n}{4}\right\rceil$. He proved that the bound in the conjecture is best possible. He also proposed a more detailed conjecture that holds all the open cases in his main conjecture.

\subsubsection{Sierpiński graphs}

The case of the well known Sierpiński graphs (see Figure 10) is dealt with by Dorbec and Klavžar in [12]. Exact values are given for all Sierpiński graphs, for power domination or generalized power domination as described in Section 5.

Another related family called WK-pyramidal networks was studied by Varghese and Vijayakumar in [28]. This family contains Sierpiński graphs as an induced sub- 
graph, with the addition of (pyramidal) extra vertices in each clique (see Figure 11). Again, the generalized power domination number of almost all WK-pyramidal networks is explicitely given.

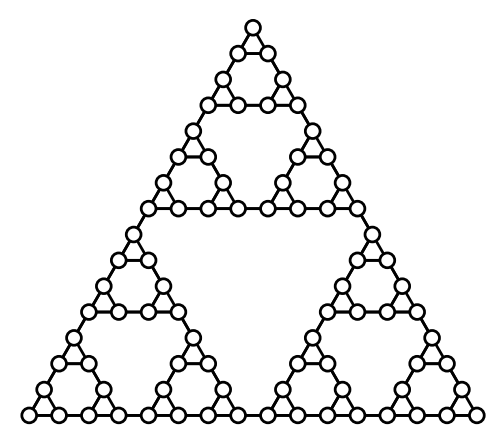

Fig. 10 The Sierpiński graph $S_{3}^{4}$

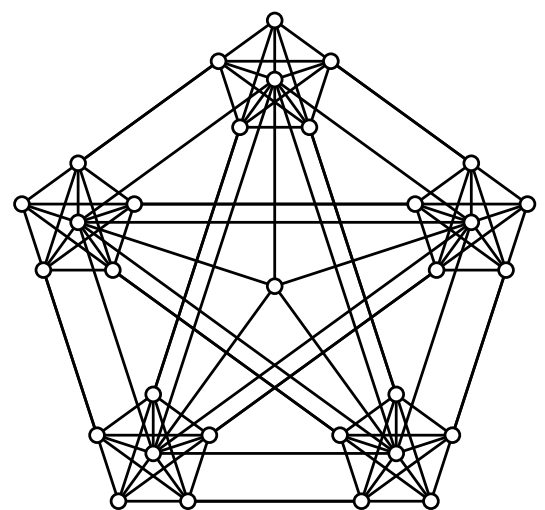

Fig. 11 The WK-pyramidal network $W K P_{(5,2)}$.

\subsubsection{Other families}

We here present the other families which were considered, without much details. For De Bruijn graphs and Kautz graphs, upper bounds on the power domination number were first given by Kuo and $\mathrm{Wu}$ in [23], then the exact values were characterized by Grigorious, Kalinowski and Stephen in [19].

The case of Knödel (see Figure 12) and Hanoi graphs (see Figure 13)was considered by Varghese, Vijayakumar and Hinz in [29], who gave close formulas for the power domination number of subfamilies of these graphs.

\section{Generalized power domination}

A common generalization of domination and power domination was introduced by Chang et al. in [8], called $k$-power domination. In this section, we give its definition and state some of the main results on $k$-power domination. 


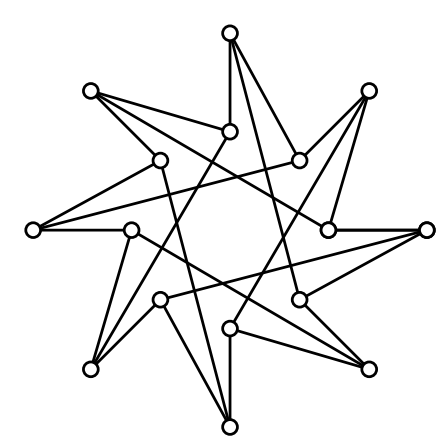

Fig. 12 The Knödel graph $W_{3,16}$

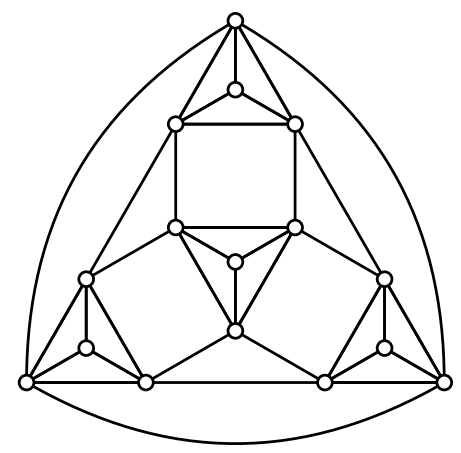

Fig. 13 The Hanoi graph $H P_{4}^{2}$.

\subsection{Definition}

The idea is to denote by $k$ the number of non-monitored neighbors of a vertex that a monitored vertex may propagate to. This gives the following definition:

\section{Definition 5 (Chang et al. [8])}

Let $G$ be a graph, $S \subseteq V(G)$ and $k$ a non-negative integer. We define the sets $\left(\mathcal{P}_{k}^{i}(S)\right)_{i \geq 0}$ of vertices monitored by $S$ at step $i$ by the following rules.

- $\mathcal{P}_{k}^{0}(S)=N[S]$.

- $\mathcal{P}_{k}^{i+1}(S)=\bigcup N[v], v \in \mathcal{P}_{k}^{i}(S)$ such that $\left|N[v] \backslash \mathcal{P}_{k}^{i}(S)\right| \leq k$.

Similarly as for power domination, a set $S$ of vertices is a $k$-power dominating set of a graph $G$ if $\mathcal{P}_{k}^{\infty}(S)=V(G)$.

Note that with this definition, $\gamma_{P, 0}(G)=\gamma(G)$ and $\gamma_{P, 1}(G)=\gamma_{P}(G)$, so we have a common generalization of domination and power domination. This may sound a bit artificial, though many results presented below tend to show that this makes sense.

\subsection{First results}

One first thing to observe about $k$-power domination is that in any graph, a $k$-power dominating set is also by definition a $(k+1)$-power dominating set. We thus naturally have

$$
\gamma(G) \geq \gamma_{P}(G) \geq \gamma_{P, 2}(G) \geq \gamma_{P, 3}(G) \geq \ldots
$$

It was noted in [8] that this inequality chain can not be improved in a general setting:

Remark 1 (Chang et al. [8]) For any finite nonincreasing sequence of positive integers $\left(x_{k}\right)_{0 \leq k \leq n}$, there exists a graph $G$ such that $\gamma_{P, k}(G)=x_{k}$ for $0 \leq k \leq n$. 
The construction to prove this statement is a generalization of the construction of the family $\mathcal{T}$ that was used to show the $\frac{n}{3}$ bound for domination. Start initially with the corona of a complete graph $K_{x_{0}}$. This enforces $\gamma(G)=x_{0}$. Then to $x_{1}$ vertices of the complete graph, attach a second leaf, and possibly link it with the previous leaf. To $x_{2}$ of these vertices, attach a third leaf and add any number of links between the three leaves. Continue in such a way until $x_{k}=1$ or the sequence is finished. See Figure 14 for an example.

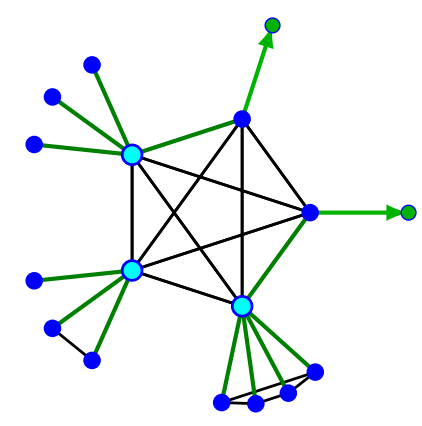

Fig. 14 An example of a graph $G$ with $\gamma(G)=5, \gamma_{P}(G)=3=\gamma_{P, 2}(G)$, and $\gamma_{P, 3}(G)=1$. A minimum (1-)power dominating set is also drawn.

\subsection{Bounds for generalized power domination}

Using this generalized setting, many results proved on power domination can be extended. We now summarize some of the main bounds found. Most of them were mentioned earlier, for the power domination number.

The first result is the following:

Theorem 14 (Chang et al. [8]) For any connected graph $G$ on $n \geq k+1$ vertices, we have $\gamma_{P, k}(G) \leq \frac{n}{k+2}$, and this bound is best possible.

This result can be proved in the same way as the $\frac{n}{3}$ bound for (1-)power domination, and the examples reaching the bound are basically the same, obtained from any graph $G$, to which each vertex is attached $k+1$ leaves.

Interestingly enough, the (1-)power domination bound for regular graphs also extends well to generalized power domination, and this is actually how the result is proved in [11].

Theorem 15 (Dorbec et al. [11])

Let $k \geq 1$ and let $G$ be a connected $(k+2)$-regular graph of order $n$. If $G \neq$ $K_{k+2, k+2}$, then $\gamma_{P, k}(G) \leq \frac{n}{k+3}$, and this bound is tight. 
It should be noted that the Sierpiński graphs in [12] and the WK-pyramidal networks in [28] are studied in the generalized setting.

\section{Propagation radius}

Another parameter closely related to power domination was introduced to indicate the number of propagation steps required to monitor the whole graph from a minimum power dominating set.

It was introduced independently first by Dorbec and Klavžar in [12], where they called the parameter propagation radius (denoted $\operatorname{rad}_{\mathrm{P}, k}(G)$ for $k$-power domination), and later by Ferrero et al. [16], who called it power propagation time (denoted $\operatorname{ppt}(G))$. We here stick to the earlier name and notation, namely the propagation radius.

Definition 6 (Propagation radius) The radius of a $k$-power dominating set $S$ of a graph $G$ is defined by

$$
\operatorname{rad}_{\mathrm{P}, k}(G, S)=1+\min \left\{i: \mathcal{P}_{k}^{i}(S)=V(G)\right\} .
$$

The $k$-propagation radius of a graph $G$ can be expressed as

$$
\operatorname{rad}_{P, k}(G)=\min \left\{\operatorname{rad}_{\mathrm{P}, k}(G, S), S \text { is a } k \text {-PDS of } G,|S|=\gamma_{P, k}(G)\right\} \text {. }
$$

Note that the power propagation time, denoted $\operatorname{ppt}(G)$, is defined so that it exactly matches the propagation radius, and we have $\operatorname{ppt}(G)=\operatorname{rad}_{\mathrm{P}, 1}(G)$. Note though that things are made a little confusing due to a slight difference between the notations of Aazami [1] and Chang et al. [8] for monitored sets at step $i$. In the first paper, $N[S]$ is the step 1 of monitoring (denoted $N[S]=S^{[1]}$ in [16]), while in the second, $N[S]=\mathcal{P}^{0}(S)$. Fortunately, the values coincide for the propagation radius.

The following early results are proved for the propagation radius:

Theorem 16 (Dorbec, Klavžar [12]) Let $G$ be a graph and $k$ a positive integer.

- We have $\gamma_{P}(G)=\gamma(G)$ if and only if $\operatorname{rad}_{P, 1}(G)=1$.

- If $\Delta(G) \leq k+1$, then $\gamma_{P, k}(G)=1$ and $\operatorname{rad}_{P, k}(G)=\operatorname{rad}(G)$.

It should be noted that $\gamma_{P, k}(G)=1$ implies $\operatorname{rad}_{\mathrm{P}, k}(G) \geq \operatorname{rad}(G)$ but not the equality, as is illustrated by the graph of Figure 15.

From the propagation radius, we can also infer a bound on the power domination number with the number of peripheral vertices. Since the number of peripheral vertices may not increase in 1-power domination, and these are the only vertices that can propagate, the peripheral vertices invariant translates to the following result:

Theorem 17 (Liao [24]) For any graph $G$ on $n$ vertices with maximum degree $\Delta$, we have:

$$
\gamma_{P}(G) \geq \frac{n}{1+\Delta \operatorname{rad}_{P, 1}(G)}
$$




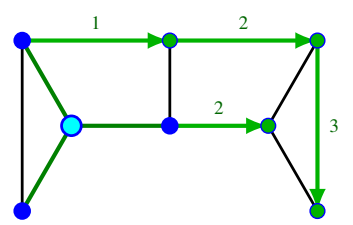

Fig. 15 In the above graph with $\gamma_{P}(G)=1, \operatorname{rad}(G)=2$ but $\operatorname{rad}_{P, 1}(G)=4$

This is easily verified by the fact that the number of peripheral vertices at all time is at most $\Delta \gamma_{P}(G)$, so the total number of vertices monitored is at most

$$
\mathcal{P}^{\infty}(S) \leq \gamma_{P}(G)+\Delta \gamma_{P}(G) \operatorname{rad}_{P, 1}(G)
$$

On its own, this result is difficult to use since there is no general relationship between the diameter or the radius of a graph and its power domination radius, as observed by Dorbec and Klavžar [12] and independently by Ferrero et al. [16]. Similar examples were given of graphs with small power domination number, small diameter and very large propagation radius. One such example from [12] is depicted in Figure 16. It illustrates the statement for 3-power domination.

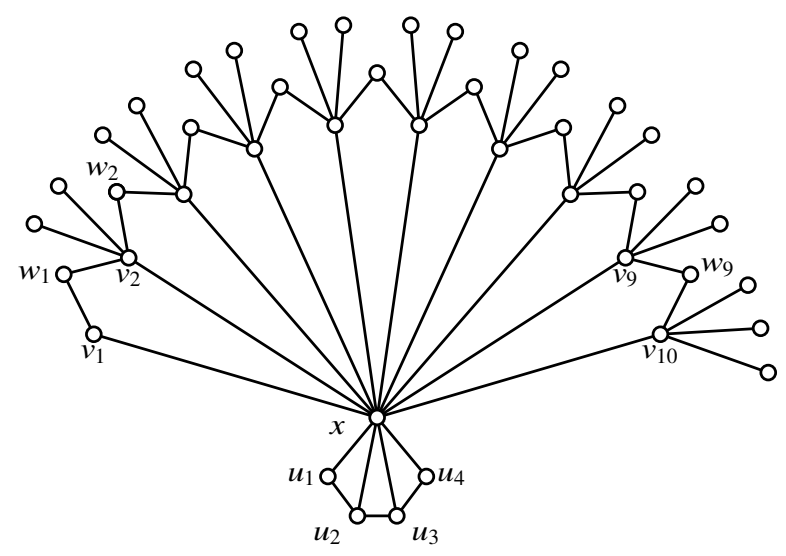

Fig. 16 The peacock graph, with 3-power domination number 1, radius 2, but 3-propagation radius equal to 11 .

The number of articles where the propagation radius has been studied is not yet very large. In the initial paper [12], the propagation radius of Sierpiński graphs was computed in the same time as their $k$-power domination number. In [14], in the same time as the authors studied the changes on the generalized power domination number by canonical graph changes, they considered the possible evolution of the propagation radius (when the power domination number was modified). In [28], Varghese and 
Vijayakumar considered the propagation radius for WK-pyramidal networks, while in [29], Varghese, Vijayakumar and Hinz studied the power domination number and propagation radius in Knödel graphs and Hanoi graphs.

\section{Open problems and perspectives}

In this final section, we present some research directions and questions that arise from the results surveyed here.

\subsection{About the relationship between $k$ and $\ell$-power domination}

One first question is about the link between the $k$ and $\ell$-power domination numbers of a graph for $k \neq \ell$. It was stated in Section 5 that when $k$ increases, the $k$ power domination number can only decrease. We have shown how these domination numbers can vary quite freely, but these examples use articulation points (i.e. vertices whose removal disconnect the graph). The following questions come naturally, with a special interest in 2-connected graphs to avoid all the cases using articulation points.

\section{? Questions}

- Can we find a characterization of the 2-connected graphs such that $\gamma_{P, k}(G)=$ $\gamma_{P, \ell}(G)$ for some $k<\ell$ ? And in particular for $\ell=k+1$ ?

- Can we answer the same question in general?

\subsection{About regular graphs}

For regular graphs, a little is known already, and more could probably be proved. Everything that is known for power domination extends nicely to generalized power domination, and is summarized as follow. For a connected regular graph $G$ of order $n$ and degree $\Delta$, we have:

- If $\Delta \leq k+1, \gamma_{P, k}(G)=1$.

- If $\Delta=k+2, \gamma_{P, k}(G) \leq \frac{n}{\Delta+1}$, with the single exception of the bipartite complete graph $K_{k+2, k+2}$.

A conjecture was made in [11] that the bound $\frac{n}{\Delta+1}$ could hold for larger $\Delta$, independently of $k$. Seemingly, some counterexamples were found to that conjecture 
at a workshop in Balatonalmádi in 2017, though there is no writen evidence. The following question of an upper bound certainly is of interest, then.

\section{? Questions}

- What is the best possible upper bound on the power domination number of a connected regular graph with degree at least four?

- More generally, what is the best possible upper bound on the $k$-power domination number of a connected regular graph with degree at least $k+3$ ?

\subsection{Hypercubes and products}

Domination in graph products is a nice but difficult problem, as illustrated by Vizing's conjecture. However, power domination seems to behave quite differently on products, as we have seen in Section 4.1.

First, the question of the hypercube is still open. The intuition that $\gamma_{P}\left(Q_{i+1}\right)$ would be equal to $\gamma\left(Q_{i}\right)$ for all $i$ was disproved, with the smallest counterexample being the pair $Q_{5}-Q_{6}$. The question remains open for generalized power domination, as mentioned below. We also present a general question for graph products.

\section{? Questions}

- Can more counterexamples be found to disprove that $\gamma_{P, k+1}\left(Q_{i+1}\right)=\gamma_{P, k}\left(Q_{i}\right)$ for $k \geq 1$ ? Can we predict the smallest counter-example for a given $k$ ?

- For some product $\otimes$, can we find some nontrivial way to relate $\gamma_{P, k}(G), \gamma_{P, \ell}(H)$ and some $\gamma_{P, f(k, \ell)}(G \otimes H)$ ? Can such relations be completed with relations also on the propagation radii?

\subsection{Maximal planar graphs}

For planar graphs, we surveyed the known results in Section 3.3. The problems sound interesting and promising, but the initial problem remains open, with the following questions.

\section{? Questions}


- Is there an infinite maximal planar graph family that requires more than $\frac{n}{6}$ vertices to power dominate, as does the triakis-tetrahedron of Figure 7 ?

- More generally, what is the best $\alpha$ such that for all maximal planar graphs $G$, $\gamma_{P}(G) \leq \alpha|V|+O(1)$ ?

- Finally, what is the best possible upper bound on the size of a minimum $k$-power dominating set of a maximal planar graph?

Note that the second question is likely to be difficult, as the corresponding question for domination remains open.

\subsection{Propagation radius}

To conclude, there are many other questions to explore concerning graph classes, in particular, the introduction of the propagation radius opens up many new questions.

\section{? Question}

- For the main results proposed up to now on power domination, what can be said about the propagation radius?

\section{References}

1. A. Aazami. Domination in graphs with bounded propagation: algorithms, formulations and hardness results. J. Comb. Optim., 19(4):429-456, 2010.

2. AIM Minimum Rank Group. Zero forcing sets and the minimum rank of graphs. Linear Algebra and its Applications, 428(7):1628 - 1648, 2008.

3. T. L. Baldwin, L. Mili, M. B. Boisen, and R. Adapa. Power system observability with minimal phasor measurement placement. IEEE Transactions on Power Systems, 8(2):707-715, 1993.

4. R. Barrera and D. Ferrero. Power domination in cylinders, tori, and generalized Petersen graphs. Networks, 58(1):43-49, 2011.

5. K. F. Benson, D. Ferrero, M. Flagg, V. Furst, L. Hogben, V. Vasilevska, and B. Wissman. Zero forcing and power domination for graph products. Australas. J. Combin., 70:221-235, 2018.

6. P. Bose, C. Pennarun, and S. Verdonschot. Power domination on triangular grids. CoRR, abs/1707.02760, 2017.

7. B. Brešar, C. Bujtás, T. Gologranc, S. Klavžar, G. Košmrlj, B. Patkós, Z. Tuza, and M. Vizer. Grundy dominating sequences and zero forcing sets. Discrete Optim., 26:66-77, 2017.

8. G. J. Chang, P. Dorbec, M. Montassier, and A. Raspaud. Generalized power domination of graphs. Discrete Appl. Math., 160(12):1691-1698, 2012. 
9. N. Dean, A. Ilic, I. Ramírez, J. Shen, and K. Tian. On the power dominating sets of hypercubes. In 14th IEEE International Conference on Computational Science and Engineering, CSE 2011, Dalian, China, August 24-26, 2011, pages 488-491, 2011.

10. P. Dorbec, A. González, and C. Pennarun. Power domination in maximal planar graphs. http://arxiv.org/abs/1706.10047, 2017.

11. P. Dorbec, M. A. Henning, C. Löwenstein, M. Montassier, and A. Raspaud. Generalized power domination in regular graphs. SIAM J. Discrete Math., 27(3):1559-1574, 2013.

12. P. Dorbec and S. Klavžar. Generalized power domination: propagation radius and Sierpiński graphs. Acta Appl. Math., 134:75-86, 2014.

13. P. Dorbec, M. Mollard, S. Klavžar, and S. Špacapan. Power domination in product graphs. SIAM J. Discrete Math., 22(2):554-567, 2008

14. P. Dorbec, S. Varghese, and A. Vijayakumar. Heredity for generalized power domination. Discrete Math. Theor. Comput. Sci., 18(3):Paper No. 5, 11, Apr. 2016.

15. M. Dorfling and M. A. Henning. A note on power domination in grid graphs. Discrete Appl. Math., 154(6):1023-1027, 2006.

16. D. Ferrero, L. Hogben, F. H. J. Kenter, and M. Young. Note on power propagation time and lower bounds for the power domination number. J. Comb. Optim., 34(3):736-741, 2017.

17. D. Ferrero, L. Hogben, F. H. J. Kenter, and M. Young. The relationship between $k$-forcing and $k$-power domination. Discrete Math., 341(6):1789-1797, 2018.

18. D. Ferrero, S. Varghese, and A. Vijayakumar. Power domination in honeycomb networks. $J$. Discrete Math. Sci. Cryptogr., 14(6):521-529, 2011.

19. C. Grigorious, T. Kalinowski, and S. Stephen. On the power domination number of de Bruijn and Kautz digraphs. In Combinatorial algorithms, volume 10765 of Lecture Notes in Comput. Sci., pages 264-272. Springer, Cham, 2018.

20. T. W. Haynes, S. M. Hedetniemi, S. T. Hedetniemi, and M. A. Henning. Domination in graphs applied to electric power networks. SIAM J. Discrete Math., 15(4):519-529, 2002.

21. L. Kang and N. Wormald. Minimum power dominating sets of random cubic graphs. J. Graph Theory, 85(1):152-171, 2017.

22. K. M. Koh and K. W. Soh. Power domination of the cartesian product of graphs. AKCE Int. J. Graphs Comb., 13(1):22-30, 2016.

23. J. Kuo and W.-L. Wu. Power domination in generalized undirected de Bruijn graphs and Kautz graphs. Discrete Math. Algorithms Appl., 7(1):1550003, 8, 2015.

24. C.-S. Liao. Power domination with bounded time constraints. J. Comb. Optim., 31(2):725-742, 2016.

25. K.-J. Pai and W.-J. Chiu. A note on âĂIJon the power dominating sets of hypercubesâĂİ. In The 29th Workshop on Combinatorial Mathematics and Computation Theory, 2012.

26. K. W. Soh and K. M. Koh. A note on power domination problem in diameter two graphs. AKCE Int. J. Graphs Comb., 11(1):51-55, 2014.

27. K. W. Soh and K. M. Koh. Recent results on the power domination numbers of graph products. New Zealand J. Math., 48:41-53, 2018.

28. S. Varghese and A. Vijayakumar. Generalized power domination in WK-pyramid networks. Bull. Inst. Combin. Appl., 78:52-68, 2016.

29. S. Varghese, A. Vijayakumar, and A. M. Hinz. Power domination in Knödel graphs and Hanoi graphs. Discuss. Math. Graph Theory, 38(1):63-74, 2018.

30. S. Wilson. Power domination on permutation graphs. Discrete Appl. Math., 262:169-178, 2019.

31. G. Xu and L. Kang. On the power domination number of the generalized Petersen graphs. $J$. Comb. Optim., 22(2):282-291, 2011.

32. M. Zhao, L. Kang, and G. J. Chang. Power domination in graphs. Discrete Math., 306(15):1812-1816, 2006. 